\title{
Some New Theorems on $c$-Distance without Continuity in Cone Metric Spaces over Banach Algebras
}

\author{
Yan Han $\mathbb{D}^{1}$ and Shaoyuan $\mathrm{Xu} \mathbb{i D}^{2}$ \\ ${ }^{1}$ School of Mathematics and Statistics, Zhaotong University, Zhaotong 657000, China \\ ${ }^{2}$ School of Mathematics and Statistics, Hanshan Normal University, Chaozhou 521041, China \\ Correspondence should be addressed to Yan Han; hanyan702@126.com
}

Received 25 February 2018; Accepted 8 April 2018; Published 15 May 2018

Academic Editor: Pasquale Vetro

Copyright (c) 2018 Yan Han and Shaoyuan Xu. This is an open access article distributed under the Creative Commons Attribution License, which permits unrestricted use, distribution, and reproduction in any medium, provided the original work is properly cited.

The fixed point theorems for one mapping and the common fixed point theorems for two mappings satisfying generalized Lipschitz conditions are obtained, without appealing to continuity for mappings or normality for cone in the conditions. Furthermore, we not only get the existence of the fixed point but also get the uniqueness. These results greatly improve and generalize several well-known comparable results in the literature. Moreover, example is given to support our new results.

\section{Introduction and Preliminaries}

The cone metric space was initiated in 2007 by Huang and Zhang [1] as a generalization of metric space. Then, many fixed point results in cone metric spaces were introduced in [2-4] and references mentioned therein. However, it is not popular since some authors have appealed to the equivalence between some fixed point results in metric spaces and in (topological vector spaces valued) cone metric spaces. Afterwards, Liu and $\mathrm{Xu}$ [5] firstly defined cone metric space over Banach algebra and obtained some fixed point theorems in such spaces. Moreover, they gave an example to illustrate the nonequivalence of version of fixed point theorems between cone metric spaces over Banach algebras and metric spaces (in usual sense). In 2011, Cho et al. [6] and Wang and Guo [7] defined the concept of $c$-distance which is a cone version of $w$ distance of Kada et al. [8]. After that, lots of fixed point results on $c$-distance in cone metric spaces and in tvs-cone metric spaces were introduced in [9-17]. However, the conditions relied strongly on the assumptions that the underlying cone is normal or the mappings are continuous.

In this paper, by exploiting the assumption of normality of the cone and the notion of continuity of the mappings at the same times, we establish some fixed point theorems on $c$-distance in cone metric spaces over Banach algebras.
Our main results improve and generalize some important known results in the literature [6, 7, 9-17]. In addition, we give an example to show that the main results are indeed real improvements and generalizations of the corresponding results in the literature.

First, we recall some basic terms and definitions about Banach algebras and cone metric spaces.

Let $\mathscr{A}$ be a real Banach algebra; that is, $\mathscr{A}$ is a real Banach space in which an operation of multiplication is defined, subject to the following properties: for all $x, y, z \in \mathscr{A}, a \in \mathbb{R}$,

(1) $x(y z)=(x y) z$

(2) $x(y+z)=x y+x z$ and $(x+y) z=x z+y z$;

(3) $a(x y)=(a x) y=x(a y)$;

(4) $\|x y\| \leq\|x\|\|y\|$.

In this paper, we shall assume that the Banach algebra $\mathscr{A}$ has a unit (i.e., a multiplicative identity) $e$ such that $e x=x e=$ $x$ for all $x \in \mathscr{A}$. An element $x \in \mathscr{A}$ is said to be invertible if there is an inverse element $y \in \mathscr{A}$ such that $x y=y x=e$. The inverse of $x$ is denoted by $x^{-1}$. For more details, we refer to [18].

The following proposition is well-known (see $[5,18])$. 
Proposition 1. Let $\mathscr{A}$ be a real Banach algebra with a unit e and $x \in \mathscr{A}$. If the spectral radius $r(x)$ of $x$ is less than 1 , that is,

$$
r(x)=\lim _{n \rightarrow \infty}\left\|x^{n}\right\|^{1 / n}=\inf _{n \geq 1}\left\|x^{n}\right\|^{1 / n}<1,
$$

then $e-x$ is invertible. Actually,

$$
(e-x)^{-1}=\sum_{i=0}^{\infty} x^{i} .
$$

A subset $P$ of $\mathscr{A}$ is called a cone if

(i) $P$ is nonempty, closed, and $\{\theta, e\} \subset P$, where $\theta$ denotes the zero element of $\mathscr{A}$;

(ii) $\alpha P+\beta P \in P$ for all nonnegative real numbers $\alpha$, $\beta$;

(iii) $P^{2}=P P \subset P$;

(iv) $P \cap(-P)=\{\theta\}$.

For a given cone $P \subset \mathscr{A}$, we can define a partial ordering $\preceq$ with respect to $P$ by $x \preceq y$ if and only if $y-x \in P$. We shall write $x \prec y$ if $x \preceq y$ and $x \neq y$, while $x \ll y$ will stand for $y-x \in$ int $P$, where int $P$ denotes the interior of $P$.

A cone $P$ is called normal if there is a number $K>0$ such that, for all $x, y \in \mathscr{A}$,

$$
\theta \preceq x \preceq y \text { implies }\|x\| \leq K\|y\| .
$$

The least positive number satisfying the above inequality is called the normal constant of $P$.

In the following, we always assume that $P$ is a cone in Banach algebra $\mathscr{A}$ with int $P \neq \emptyset$ and $\preceq$ is the partial ordering with respect to $P$.

Definition 2 (see $[1,5])$. Let $X$ be a nonempty set. Suppose that the mapping $d: X \times X \rightarrow \mathscr{A}$ satisfies

(d1) $\theta \preceq d(x, y)$ for all $x, y \in X$ and $d(x, y)=\theta$ if and only if $x=y$;

(d2) $d(x, y)=d(y, x)$ for all $x, y \in X$;

(d3) $d(x, y) \preceq d(x, z)+d(z, y)$ for all $x, y, z \in X$.

Then $d$ is called a cone metric on $X$ and $(X, d)$ is called a cone metric space over a Banach algebra $\mathscr{A}$.

Definition 3 (see $[1,5])$. Let $(X, d)$ be a cone metric space over a Banach algebra $\mathscr{A}, x \in X$ and $\left\{x_{n}\right\}$ a sequence in $X$. Then we say that is

(i) $\left\{x_{n}\right\}$ converges to $x$ if for every $c \in \mathscr{A}$ with $c \gg \theta$, there is a natural number $N$ such that for all $n>N$, $d\left(x_{n}, x\right) \ll c ;$

(ii) $\left\{x_{n}\right\}$ is a Cauchy sequence if for every $c \in \mathscr{A}$ with $c \gg$ $\theta$, there is a natural number $N$ such that for all $n, m>$ $N, d\left(x_{n}, x_{m}\right) \ll c ;$

(iii) $(X, d)$ is a complete cone metric space if every Cauchy sequence is convergent in $X$.

Definition 4 (see $[6,7,13])$. Let $(X, d)$ be a cone metric space over a Banach algebra $\mathscr{A}$. Then the mapping $q: X \times X \rightarrow \mathscr{A}$ is called a $c$-distance on $X$ if the following are satisfied: (q1) $\theta \preceq q(x, y)$ for all $x, y \in X$

(q2) $q(x, y) \preceq q(x, z)+q(z, y)$ for all $x, y, z \in X$.

(q3) For each $x \in X$ and $n \geq 1$, if $q\left(x, y_{n}\right) \preceq u$ for some $u=u_{x} \in P$, then $q(x, y) \preceq u$ whenever $\left\{y_{n}\right\}$ is a sequence in $X$ converging to a point $y \in X$.

(q4) For all $c \in \mathscr{A}$ with $\theta \ll c$, there exists $e \in \mathscr{A}$ such that $q(z, x) \ll e$, and $q(z, y) \ll e$ imply $d(x, y) \ll c$.

Remark 5 (see [7]). The following facts are well-known:

(1) $q(x, y)=q(y, x)$ does not necessarily hold for all $x, y \in X$.

(2) $q(x, y)=\theta$ is not necessarily equivalent to $x=y$ for all $x, y \in X$.

(3) The $c$-distance is a great generalization of the $w$ distance.

(4) If $q(x, y)=d(x, y)$, then $q$ is a $c$-distance. That is, $c$ distance is also a generalization of cone metric.

Lemma 6 (see [2]). The limit of a convergent sequence in cone metric space is unique.

Lemma 7 (see [6]). Let $(X, d)$ be a cone metric space and $q$ be a c-distance on $X$. Let $\left\{x_{n}\right\}$ and $\left\{y_{n}\right\}$ be sequences in $X$ and $x, y, z \in X$. Suppose that $\left\{u_{n}\right\}$ is a sequence in $P$ converging to $\theta$. Then the following hold:

(1) If $q\left(x_{n}, y\right) \preceq u_{n}$ and $q\left(x_{n}, z\right) \preceq u_{n}$, then $y=z$.

(2) If $q\left(x_{n}, y_{n}\right) \preceq u_{n}$ and $q\left(x_{n}, z\right) \preceq u_{n}$, then $\left\{y_{n}\right\}$ converges to $z$.

(3) If $q\left(x_{n}, x_{m}\right) \preceq u_{n}$ for $m>n$, then $\left\{x_{n}\right\}$ is a Cauchy sequence in $X$.

(4) If $q\left(y, x_{n}\right) \preceq u_{n}$, then $\left\{x_{n}\right\}$ is a Cauchy sequence in $X$.

Lemma 8 (see [12]). Let $(X, d)$ be a cone metric space and $q$ be a $c$-distance on $X$. Let $\left\{x_{n}\right\}$ be a sequence in $X$. Suppose that $\left\{\alpha_{n}\right\}$ and $\left\{\beta_{n}\right\}$ are two sequences in $P$ converging to $\theta$. If $q\left(x_{n}, y\right) \preceq \alpha_{n}$ and $q\left(x_{n}, z\right) \preceq \beta_{n}$, then $y=z$.

Lemma 9 (see $[13])$. Let $(X, d)$ be a cone metric space and $q$ be a c-distance on $X$. If $x, y \in X$ such that $q(x, y)=\theta$ and $q(y, x)=\theta$, then $x=y$.

Lemma 10 (see [18]). Let $\mathscr{A}$ be a Banach algebra with a unit e. If $x, y \in \mathscr{A}$, and $x$ commutes with $y$, then

$$
\begin{gathered}
r(x+y) \leq r(x)+r(y), \\
r(x y) \leq r(x) r(y)
\end{gathered}
$$

Lemma 11 (see [19]). Let $\mathscr{A}$ be a Banach algebra with a unit $e$ and let $k$ be a vector in $\mathscr{A}$. If $0 \leq r(k)<1$, then we have $r\left((e-k)^{-1}\right) \leq(1-r(k))^{-1}$.

Lemma 12 (see [20]). Let $\mathscr{A}$ be a Banach algebra with a unit $e$ and $P$ be a solid cone in $\mathscr{A}$. Let $u, \alpha, \beta \in P$ hold $\alpha \preceq \beta$ and $u \preceq \alpha u$. If $r(\beta)<1$, then $u=\theta$. 


\section{Main Results}

In this section, without the assumption of normality of the cone and the notion of continuity of the mappings at the same times, we prove some fixed point theorems for $c$-distance in the setting of cone metric spaces over Banach algebras. All conclusions are new. Moreover, we illustrate our results by an example.

Theorem 13. Let $(X, d)$ be a complete cone metric space over Banach algebra $\mathscr{A}$ and the underlying solid cone $P$. Let $q$ be $a$ $c$-distance on $X$. Suppose the mapping $f: X \rightarrow X$ satisfies generalized Lipschitz conditions:

$$
\begin{aligned}
q(f x, f y) \leq & a_{1} q(x, y)+a_{2} q(x, f x)+a_{3} q(x, f y) \\
& +a_{4} q(f x, y) \\
q(f y, f x) \leq & a_{1} q(y, x)+a_{2} q(f x, x)+a_{3} q(f y, x) \\
& +a_{4} q(y, f x),
\end{aligned}
$$

for all $x, y \in X$, where $a_{i} \in P(i=1,2,3,4)$ are generalized Lipschitz constants with $r\left(a_{3}\right)+r\left(a_{1}+a_{2}+a_{3}+2 a_{4}\right)<1$. If $a_{3}$ commutes with $a_{1}+a_{2}+a_{3}+2 a_{4}$, then $f$ has a unique fixed point.

Proof. Suppose $x_{0}$ is an arbitrary point in $X$ and set $x_{n}=$ $f x_{n-1}=f^{n} x_{0}, n \geq 1$. According to (5), we have

$$
\begin{aligned}
q\left(x_{n}, x_{n+1}\right)= & q\left(f x_{n-1}, f x_{n}\right) \\
\preceq & a_{1} q\left(x_{n-1}, x_{n}\right)+a_{2} q\left(x_{n-1}, f x_{n-1}\right) \\
& +a_{3} q\left(x_{n-1}, f x_{n}\right)+a_{4} q\left(f x_{n-1}, x_{n}\right) \\
= & a_{1} q\left(x_{n-1}, x_{n}\right)+a_{2} q\left(x_{n-1}, x_{n}\right) \\
& +a_{3} q\left(x_{n-1}, x_{n+1}\right)+a_{4} q\left(x_{n}, x_{n}\right) \\
\leq & a_{1} q\left(x_{n-1}, x_{n}\right)+a_{2} q\left(x_{n-1}, x_{n}\right) \\
& +a_{3}\left[q\left(x_{n-1}, x_{n}\right)+q\left(x_{n}, x_{n+1}\right)\right] \\
& +a_{4}\left[q\left(x_{n}, x_{n-1}\right)+q\left(x_{n-1}, x_{n}\right)\right]
\end{aligned}
$$

which means that

$$
\begin{aligned}
\left(e-a_{3}\right) q\left(x_{n}, x_{n+1}\right) \preceq & \left(a_{1}+a_{2}+a_{3}+a_{4}\right) q\left(x_{n-1}, x_{n}\right) \\
& +a_{4} q\left(x_{n}, x_{n-1}\right) .
\end{aligned}
$$

Making full use of (6), we obtain

$$
\begin{aligned}
q\left(x_{n+1}, x_{n}\right)= & q\left(f x_{n}, f x_{n-1}\right) \\
\leq & a_{1} q\left(x_{n}, x_{n-1}\right)+a_{2} q\left(f x_{n-1}, x_{n-1}\right) \\
& +a_{3} q\left(f x_{n}, x_{n-1}\right)+a_{4} q\left(x_{n}, f x_{n-1}\right) \\
= & a_{1} q\left(x_{n}, x_{n-1}\right)+a_{2} q\left(x_{n}, x_{n-1}\right) \\
& +a_{3} q\left(x_{n+1}, x_{n-1}\right)+a_{4} q\left(x_{n}, x_{n}\right)
\end{aligned}
$$

$$
\begin{aligned}
\preceq & a_{1} q\left(x_{n}, x_{n-1}\right)+a_{2} q\left(x_{n}, x_{n-1}\right) \\
& +a_{3}\left[q\left(x_{n+1}, x_{n}\right)+q\left(x_{n}, x_{n-1}\right)\right] \\
& +a_{4}\left[q\left(x_{n}, x_{n-1}\right)+q\left(x_{n-1}, x_{n}\right)\right],
\end{aligned}
$$

which implies that

$$
\begin{aligned}
\left(e-a_{3}\right) q\left(x_{n+1}, x_{n}\right) \preceq & \left(a_{1}+a_{2}+a_{3}+a_{4}\right) q\left(x_{n}, x_{n-1}\right) \\
& +a_{4} q\left(x_{n-1}, x_{n}\right) .
\end{aligned}
$$

Thus, we can find the sum of (8) and (10) as follows:

$$
\begin{aligned}
& \left(e-a_{3}\right)\left[q\left(x_{n}, x_{n+1}\right)+q\left(x_{n+1}, x_{n}\right)\right] \\
& \quad \preceq\left(a_{1}+a_{2}+a_{3}+2 a_{4}\right)\left[q\left(x_{n-1}, x_{n}\right)+q\left(x_{n}, x_{n-1}\right)\right] .
\end{aligned}
$$

Further, we set $u_{n}=q\left(x_{n}, x_{n+1}\right)+q\left(x_{n+1}, x_{n}\right)$. Then, we see

$$
\left(e-a_{3}\right) u_{n} \preceq\left(a_{1}+a_{2}+a_{3}+2 a_{4}\right) u_{n-1} \text {. }
$$

Since $r\left(a_{3}\right)<r\left(a_{3}\right)+r\left(a_{1}+a_{2}+a_{3}+2 a_{4}\right)<1$, then, by Proposition 1 , it is easy to see that $e-a_{3}$ is invertible. Furthermore,

$$
\left(e-a_{3}\right)^{-1}=\sum_{i=0}^{\infty} a_{3}^{i} .
$$

Let $h=\left(e-a_{3}\right)^{-1}\left(a_{1}+a_{2}+a_{3}+2 a_{4}\right)$. As $a_{3}$ commutes with $a_{1}+a_{2}+a_{3}+2 a_{4}$, it follows that

$$
\begin{aligned}
\left(e-a_{3}\right)^{-1}\left(a_{1}+a_{2}+a_{3}+2 a_{4}\right) \\
=\left(\sum_{i=0}^{\infty} a_{3}^{i}\right)\left(a_{1}+a_{2}+a_{3}+2 a_{4}\right) \\
=\left(a_{1}+a_{2}+a_{3}+2 a_{4}\right)\left(\sum_{i=0}^{\infty} a_{3}^{i}\right) \\
=\left(a_{1}+a_{2}+a_{3}+2 a_{4}\right)\left(e-a_{3}\right)^{-1},
\end{aligned}
$$

that is to say $\left(e-a_{3}\right)^{-1}$ commutes with $a_{1}+a_{2}+a_{3}+2 a_{4}$. Then by Lemmas 10 and 11, we obtain

$$
\begin{aligned}
r(h) & =r\left(\left(e-a_{3}\right)^{-1}\left(a_{1}+a_{2}+a_{3}+2 a_{4}\right)\right) \\
& \leq r\left(\left(e-a_{3}\right)^{-1}\right) r\left(a_{1}+a_{2}+a_{3}+2 a_{4}\right) \\
& \leq\left(1-r\left(a_{3}\right)\right)^{-1} r\left(a_{1}+a_{2}+a_{3}+2 a_{4}\right) \\
& =\frac{1}{1-r\left(a_{3}\right)} r\left(a_{1}+a_{2}+a_{3}+2 a_{4}\right)<1,
\end{aligned}
$$

which means that $(e-h)^{-1}=\sum_{i=0}^{\infty} h^{i}$ and $\left\|h^{n}\right\| \rightarrow 0$ as $n \rightarrow \infty$.

By multiplying in both sides of (12) by $\left(e-a_{3}\right)^{-1}$, we get

$$
\begin{aligned}
q\left(x_{n}, x_{n+1}\right) & \preceq u_{n} \\
& \preceq\left(e-a_{3}\right)^{-1}\left(a_{1}+a_{2}+a_{3}+2 a_{4}\right) u_{n-1} \\
& =h u_{n-1} \preceq \cdots \preceq h^{n} u_{0} .
\end{aligned}
$$


Let $m>n \geq 1$. We infer

$$
\begin{aligned}
q\left(x_{n}, x_{m}\right) \preceq & q\left(x_{n}, x_{n+1}\right)+q\left(x_{n+1}, x_{n+2}\right)+\cdots \\
& +q\left(x_{m-1}, x_{m}\right) \\
\preceq & \left(h^{n}+h^{n+1}+\cdots+h^{m-1}\right) u_{0} \\
= & \left(e+h+\cdots+h^{m-n-1}\right) h^{n} u_{0} \\
& \left(\sum_{i=0}^{\infty} h^{i}\right) h^{n} u_{0}=(e-h)^{-1} h^{n} u_{0} .
\end{aligned}
$$

Owing to $\left\|(e-h)^{-1} h^{n} u_{0}\right\| \leq\left\|(e-h)^{-1}\right\|\left\|h^{n}\right\|\left\|u_{0}\right\| \rightarrow 0(n \rightarrow$ $\infty)$, it leads to $(e-h)^{-1} h^{n} u_{0} \rightarrow \theta(n \rightarrow \infty)$. By Lemma 7(3), $\left\{x_{n}\right\}$ is a Cauchy sequence in $(X, d)$.

Since $X$ is complete, there exists $u \in X$ such that $x_{n}=$ $f x_{n-1} \rightarrow u$ as $n \rightarrow \infty$. By Definition 4(q3), we obtain

$$
q\left(x_{n}, u\right) \preceq(e-h)^{-1} h^{n} u_{0} .
$$

Now, we show that $f u=u$. Substituting $x=x_{n-1}, y=u$ in (5), we get

$$
\begin{aligned}
q\left(x_{n}, f u\right)= & q\left(f x_{n-1}, f u\right) \\
\preceq & a_{1} q\left(x_{n-1}, u\right)+a_{2} q\left(x_{n-1}, f x_{n-1}\right) \\
& +a_{3} q\left(x_{n-1}, f u\right)+a_{4} q\left(f x_{n-1}, u\right) \\
= & a_{1} q\left(x_{n-1}, u\right)+a_{2} q\left(x_{n-1}, x_{n}\right) \\
& +a_{3} q\left(x_{n-1}, f u\right)+a_{4} q\left(x_{n}, u\right) \\
\preceq & a_{1}\left[q\left(x_{n-1}, x_{n}\right)+q\left(x_{n}, u\right)\right] \\
& +a_{2} q\left(x_{n-1}, x_{n}\right) \\
& +a_{3}\left[q\left(x_{n-1}, x_{n}\right)+q\left(x_{n}, f u\right)\right] \\
& +a_{4} q\left(x_{n}, u\right)
\end{aligned}
$$

which implies that $\left(e-a_{3}\right) q\left(x_{n}, f u\right) \preceq\left(a_{1}+a_{2}+a_{3}\right) q\left(x_{n-1}, x_{n}\right)+$ $\left(a_{1}+a_{4}\right) q\left(x_{n}, u\right)$. Since $r\left(a_{3}\right)<1, e-a_{3}$ is invertible. So, it follows immediately from (16) and (18) that

$$
\begin{aligned}
q( & \left.x_{n}, f u\right) \preceq\left(e-a_{3}\right)^{-1}\left[\left(a_{1}+a_{2}+a_{3}\right) q\left(x_{n-1}, x_{n}\right)\right. \\
& \left.+\left(a_{1}+a_{4}\right) q\left(x_{n}, u\right)\right] \preceq\left(e-a_{3}\right)^{-1} \\
& \cdot\left[\left(a_{1}+a_{2}+a_{3}\right) h^{n-1} u_{0}\right. \\
& \left.+\left(a_{1}+a_{4}\right)(e-h)^{-1} h^{n} u_{0}\right]=\left(e-a_{3}\right)^{-1} \\
& \cdot\left[\left(a_{1}+a_{2}+a_{3}\right)+\left(a_{1}+a_{4}\right)(e-h)^{-1} h\right] h^{n-1} u_{0} .
\end{aligned}
$$

Set $\alpha_{n}=(e-h)^{-1} h^{n} u_{0}$ and $\beta_{n}=\left(e-a_{3}\right)^{-1}\left[\left(a_{1}+a_{2}+a_{3}\right)+\right.$ $\left.\left(a_{1}+a_{4}\right)(e-h)^{-1} h\right] h^{n-1} u_{0}$. As $r(h)<1$ and $\left\|h^{n}\right\| \rightarrow 0(n \rightarrow$ $\infty)$, we know $\alpha_{n}, \beta_{n} \rightarrow \theta(n \rightarrow \infty)$. Thus, by (18), (20), and Lemma 8, we get that $f u=u$.
In the following we shall show the fixed point is unique. Firstly, we have to prove $q(u, u)=\theta$. Making full use of (5), we know

$$
\begin{aligned}
q(u, u)= & q(f u, f u) \\
\leq & a_{1} q(u, u)+a_{2} q(u, f u)+a_{3} q(u, f u) \\
& +a_{4} q(f u, u) \\
= & \left(a_{1}+a_{2}+a_{3}+a_{4}\right) q(u, u) .
\end{aligned}
$$

In view of $a_{1}+a_{2}+a_{3}+a_{4} \preceq a_{1}+a_{2}+a_{3}+2 a_{4}, r\left(a_{1}+a_{2}+a_{3}+2 a_{4}\right)<$ $1-r\left(a_{3}\right)<1$, and Lemma 12 , we have $q(u, u)=\theta$. Secondly, if there is another fixed point $v$, then, from (5), we get

$$
\begin{aligned}
q(v, u)= & q(f v, f u) \\
\preceq & a_{1} q(v, u)+a_{2} q(v, f v)+a_{3} q(v, f u) \\
& +a_{4} q(f v, u) \\
= & a_{1} q(v, u)+a_{2} q(v, v)+a_{3} q(v, u) \\
& +a_{4} q(v, u)
\end{aligned}
$$

which establishes that

$$
q(v, u) \preceq\left(a_{1}+a_{3}+a_{4}\right) q(v, u) .
$$

Since $a_{1}+a_{3}+a_{4} \preceq a_{1}+a_{2}+a_{3}+2 a_{4}$ and $r\left(a_{1}+a_{2}+a_{3}+2 a_{4}\right)<$ $1-r\left(a_{3}\right)<1$, it follows immediately from Lemma 12 that $q(v, u)=\theta$. Actually, by (6), we also have that

$$
q(u, v) \preceq\left(a_{1}+a_{3}+a_{4}\right) q(u, v) .
$$

Similar to the above proof, it is not difficult to obtain that $q(u, v)=\theta$. Thus, from Lemma $9, u=v$. The conclusion is true.

Corollary 14. Let $(X, d)$ be a complete cone metric space and the underlying solid cone P. Let $q$ be a c-distance on $X$. Suppose the mapping $f: X \rightarrow X$ satisfies generalized Lipschitz conditions:

$$
\begin{aligned}
q(f x, f y) \leq & a_{1} q(x, y)+a_{2} q(x, f x)+a_{3} q(x, f y) \\
& +a_{4} q(f x, y), \\
q(f y, f x) \leq & a_{1} q(y, x)+a_{2} q(f x, x)+a_{3} q(f y, x) \\
& +a_{4} q(y, f x),
\end{aligned}
$$

for all $x, y \in X$, where $a_{i} \in \mathbb{R}^{+}(i=1,2,3,4)$ and satisfies $a_{1}+a_{2}+2 a_{3}+2 a_{4}<1$. Then, $f$ has a unique fixed point.

Proof. By taking $r\left(a_{i}\right)=a_{i}$ for each $a_{i} \in \mathbb{R}^{+}$in Theorem 13, we obtain the desired result.

Corollary 15. Let $(X, d)$ be a complete cone metric space over Banach algebra $\mathscr{A}$ and the underlying solid cone $P$. Let $q$ be $a$ $c$-distance on $X$. Suppose the mapping $f: X \rightarrow X$ satisfies generalized Lipschitz condition:

$$
q(f x, f y) \preceq a_{1} q(x, y)+a_{2} q(x, f x)+a_{3} q(x, f y),
$$


for all $x, y \in X$, where $a_{i} \in P(i=1,2,3)$ are generalized Lipschitz constants with $r\left(a_{3}\right)+r\left(a_{1}+a_{2}+a_{3}\right)<1$. If $a_{3}$ commutes with $a_{1}+a_{2}+a_{3}$, then $f$ has a unique fixed point.

Proof. Similar to Theorem 13, we get the sequence $\left\{x_{n}\right\}$ as follows: $x_{n}=f x_{n-1}=f^{n} x_{0}, n \geq 1$. Then, we have

$$
\begin{aligned}
q\left(x_{n}, x_{n+1}\right)= & q\left(f x_{n-1}, f x_{n}\right) \\
\leq & a_{1} q\left(x_{n-1}, x_{n}\right)+a_{2} q\left(x_{n-1}, f x_{n-1}\right) \\
& +a_{3} q\left(x_{n-1}, f x_{n}\right) \\
= & a_{1} q\left(x_{n-1}, x_{n}\right)+a_{2} q\left(x_{n-1}, x_{n}\right) \\
& +a_{3} q\left(x_{n-1}, x_{n+1}\right) \\
\leq & a_{1} q\left(x_{n-1}, x_{n}\right)+a_{2} q\left(x_{n-1}, x_{n}\right) \\
& +a_{3}\left[q\left(x_{n-1}, x_{n}\right)+q\left(x_{n}, x_{n+1}\right)\right],
\end{aligned}
$$

which means that

$$
\left(e-a_{3}\right) q\left(x_{n}, x_{n+1}\right) \leq\left(a_{1}+a_{2}+a_{3}\right) q\left(x_{n-1}, x_{n}\right) .
$$

Since $r\left(a_{3}\right)<r\left(a_{3}\right)+r\left(a_{1}+a_{2}+a_{3}\right)<1$, then by Proposition 1 it is easy to see that $e-a_{3}$ is invertible. Furthermore,

$$
\left(e-a_{3}\right)^{-1}=\sum_{i=0}^{\infty} a_{3}^{i} .
$$

Let $h=\left(e-a_{3}\right)^{-1}\left(a_{1}+a_{2}+a_{3}\right)$. As $a_{3}$ commutes with $a_{1}+a_{2}+a_{3}$, it follows that

$$
\begin{aligned}
& \left(e-a_{3}\right)^{-1}\left(a_{1}+a_{2}+a_{3}\right)=\left(\sum_{i=0}^{\infty} a_{3}^{i}\right)\left(a_{1}+a_{2}+a_{3}\right) \\
& =\left(a_{1}+a_{2}+a_{3}\right)\left(\sum_{i=0}^{\infty} a_{3}^{i}\right) \\
& =\left(a_{1}+a_{2}+a_{3}\right)\left(e-a_{3}\right)^{-1}
\end{aligned}
$$

that is to say $\left(e-a_{3}\right)^{-1}$ commutes with $a_{1}+a_{2}+a_{3}$. Then by Lemmas 10 and 11, we obtain

$$
\begin{aligned}
r(h) & =r\left(\left(e-a_{3}\right)^{-1}\left(a_{1}+a_{2}+a_{3}\right)\right) \\
& \leq r\left(\left(e-a_{3}\right)^{-1}\right) r\left(a_{1}+a_{2}+a_{3}\right) \\
& \leq\left(1-r\left(a_{3}\right)\right)^{-1} r\left(a_{1}+a_{2}+a_{3}\right) \\
& =\frac{1}{1-r\left(a_{3}\right)} r\left(a_{1}+a_{2}+a_{3}\right)<1,
\end{aligned}
$$

which means that $(e-h)^{-1}=\sum_{i=0}^{\infty} h^{i}$ and $\left\|h^{n}\right\| \rightarrow 0$ as $n \rightarrow \infty$.

Therefore, we get

$$
\begin{aligned}
q\left(x_{n}, x_{n+1}\right) & \leq\left(e-a_{3}\right)^{-1}\left(a_{1}+a_{2}+a_{3}\right) q\left(x_{n-1}, x_{n}\right) \\
& =h q\left(x_{n-1}, x_{n}\right) \preceq \cdots \preceq h^{n} q\left(x_{0}, x_{1}\right) .
\end{aligned}
$$

Let $m>n \geq 1$. We infer

$$
\begin{aligned}
q\left(x_{n}, x_{m}\right) \leq & q\left(x_{n}, x_{n+1}\right)+q\left(x_{n+1}, x_{n+2}\right)+\cdots \\
& +q\left(x_{m-1}, x_{m}\right) \\
\leq & \left(h^{n}+h^{n+1}+\cdots+h^{m-1}\right) q\left(x_{0}, x_{1}\right) \\
= & \left(e+h+\cdots+h^{m-n-1}\right) h^{n} q\left(x_{0}, x_{1}\right) \\
\leq & \left(\sum_{i=0}^{\infty} h^{i}\right) h^{n} q\left(x_{0}, x_{1}\right) \\
= & (e-h)^{-1} h^{n} q\left(x_{0}, x_{1}\right) .
\end{aligned}
$$

Owing to $\left\|(e-h)^{-1} h^{n} q\left(x_{0}, x_{1}\right)\right\| \leq\left\|(e-h)^{-1}\right\|\left\|h^{n}\right\| \| q\left(x_{0}\right.$, $\left.x_{1}\right) \| \rightarrow 0(n \rightarrow \infty)$, it leads to $(e-h)^{-1} h^{n} q\left(x_{0}, x_{1}\right) \rightarrow \theta(n \rightarrow$ $\infty)$. By Lemma $7(3),\left\{x_{n}\right\}$ is a Cauchy sequence in $(X, d)$.

Since $X$ is complete, there exists $u \in X$ such that $x_{n}=$ $f x_{n-1} \rightarrow u$ as $n \rightarrow \infty$. By Definition 4(q3), we obtain

$$
q\left(x_{n}, u\right) \preceq(e-h)^{-1} h^{n} q\left(x_{0}, x_{1}\right) .
$$

Now, we show that $f u=u$. By the conditions in the theorem, we get

$$
\begin{aligned}
q\left(x_{n}, f u\right)= & q\left(f x_{n-1}, f u\right) \\
\leq & a_{1} q\left(x_{n-1}, u\right)+a_{2} q\left(x_{n-1}, f x_{n-1}\right) \\
& +a_{3} q\left(x_{n-1}, f u\right) \\
= & a_{1} q\left(x_{n-1}, u\right)+a_{2} q\left(x_{n-1}, x_{n}\right) \\
& +a_{3} q\left(x_{n-1}, f u\right) \\
\leq & a_{1}\left[q\left(x_{n-1}, x_{n}\right)+q\left(x_{n}, u\right)\right] \\
& +a_{2} q\left(x_{n-1}, x_{n}\right) \\
& +a_{3}\left[q\left(x_{n-1}, x_{n}\right)+q\left(x_{n}, f u\right)\right],
\end{aligned}
$$

which implies that $\left(e-a_{3}\right) q\left(x_{n}, f u\right) \preceq\left(a_{1}+a_{2}+a_{3}\right) q\left(x_{n-1}, x_{n}\right)+$ $a_{1} q\left(x_{n}, u\right)$. Since $r\left(a_{3}\right)<1, e-a_{3}$ is invertible. So,

$$
\begin{aligned}
& q\left(x_{n}, f u\right) \leq\left(e-a_{3}\right)^{-1}\left[\left(a_{1}+a_{2}+a_{3}\right) q\left(x_{n-1}, x_{n}\right)\right. \\
& \left.\quad+a_{1} q\left(x_{n}, u\right)\right] \preceq\left(e-a_{3}\right)^{-1} \\
& \cdot\left[\left(a_{1}+a_{2}+a_{3}\right) h^{n-1} q\left(x_{0}, x_{1}\right)\right. \\
& \left.\quad+a_{1}(e-h)^{-1} h^{n} q\left(x_{0}, x_{1}\right)\right]=\left(e-a_{3}\right)^{-1} \\
& \cdot\left[\left(a_{1}+a_{2}+a_{3}\right)+a_{1}(e-h)^{-1} h\right] h^{n-1} q\left(x_{0}, x_{1}\right) .
\end{aligned}
$$

Set $\alpha_{n}=(e-h)^{-1} h^{n} q\left(x_{0}, x_{1}\right)$ and $\beta_{n}=\left(e-a_{3}\right)^{-1}\left[\left(a_{1}+a_{2}+a_{3}\right)+\right.$ $\left.a_{1}(e-h)^{-1} h\right] h^{n-1} q\left(x_{0}, x_{1}\right)$. As $r(h)<1$ and $\left\|h^{n}\right\| \rightarrow 0(n \rightarrow$ $\infty)$, we know $\alpha_{n}, \beta_{n} \rightarrow \theta(n \rightarrow \infty)$. Thus, by Lemma 8 , we get that $f u=u$. Then, similar to the proof of Theorem 13, it is not difficult to obtain that $u$ is the unique fixed point of $f$. So, the conclusion is true. 
Now, we introduce some common fixed point theorems for two mappings which satisfy generalized Lipschitz conditions, without continuity of the mappings and normality of the cone.

Theorem 16. Let $(X, d)$ be a complete cone metric space over Banach algebra $\mathscr{A}$ and the underlying solid cone $P$. Let $q$ be a $c$-distance on $X$. Suppose the mappings $f, g: X \rightarrow X$ satisfy generalized Lipschitz conditions:

$$
\begin{aligned}
& q(f x, g y) \leq a_{1} q(x, y)+a_{2} q(x, f x)+a_{3} q(x, g y), \\
& q(g x, f y) \leq a_{1} q(x, y)+a_{2} q(x, g x)+a_{3} q(x, f y) .
\end{aligned}
$$

Condition (37) holds for all $x, y \in X$ and condition (38) holds for all $x, y \in X$, where $a_{i} \in P(i=1,2,3)$ are generalized Lipschitz constants with $r\left(a_{3}\right)+r\left(a_{1}+a_{2}+a_{3}\right)<1$. If $a_{3}$ commutes with $a_{1}+a_{2}+a_{3}$, then $f, g$ have a unique common fixed point.

Proof. Suppose $x_{0}$ is an arbitrary point in $X$ and set $x_{2 n+1}=$ $f x_{2 n}, x_{2 n+2}=g x_{2 n+1}, n \geq 1$. According to (37), we have

$$
\begin{aligned}
& q\left(x_{2 n+1}, x_{2 n+2}\right)=q\left(f x_{2 n}, g x_{2 n+1}\right) \\
& \leq a_{1} q\left(x_{2 n}, x_{2 n+1}\right)+a_{2} q\left(x_{2 n}, f x_{2 n}\right) \\
&+a_{3} q\left(x_{2 n}, g x_{2 n+1}\right) \\
&= a_{1} q\left(x_{2 n}, x_{2 n+1}\right)+a_{2} q\left(x_{2 n}, x_{2 n+1}\right) \\
&+a_{3} q\left(x_{2 n}, x_{2 n+2}\right) \\
& \leq a_{1} q\left(x_{2 n}, x_{2 n+1}\right)+a_{2} q\left(x_{2 n}, x_{2 n+1}\right) \\
&+a_{3}\left[q\left(x_{2 n}, x_{2 n+1}\right)+q\left(x_{2 n+1}, x_{2 n+2}\right)\right],
\end{aligned}
$$

which means that

$$
\begin{aligned}
& \left(e-a_{3}\right) q\left(x_{2 n+1}, x_{2 n+2}\right) \\
& \quad \leq\left(a_{1}+a_{2}+a_{3}\right) q\left(x_{2 n}, x_{2 n+1}\right) .
\end{aligned}
$$

Similarly by using (38), we obtain

$$
\begin{aligned}
& q\left(x_{2 n+2}, x_{2 n+3}\right)=q\left(g x_{2 n+1}, f x_{2 n+2}\right) \\
& \leq a_{1} q\left(x_{2 n+1}, x_{2 n+2}\right)+a_{2} q\left(x_{2 n+1}, g x_{2 n+1}\right) \\
&+a_{3} q\left(x_{2 n+1}, f x_{2 n+2}\right) \\
&= a_{1} q\left(x_{2 n+1}, x_{2 n+2}\right)+a_{2} q\left(x_{2 n+1}, x_{2 n+2}\right) \\
&+a_{3} q\left(x_{2 n+1}, x_{2 n+3}\right) \\
& \leq a_{1} q\left(x_{2 n+1}, x_{2 n+2}\right)+a_{2} q\left(x_{2 n+1}, x_{2 n+2}\right) \\
&+a_{3}\left[q\left(x_{2 n+1}, x_{2 n+2}\right)+q\left(x_{2 n+2}, x_{2 n+3}\right)\right],
\end{aligned}
$$

which implies that

$$
\begin{aligned}
& \left(e-a_{3}\right) q\left(x_{2 n+2}, x_{2 n+3}\right) \\
& \quad \leq\left(a_{1}+a_{2}+a_{3}\right) q\left(x_{2 n+1}, x_{2 n+2}\right) .
\end{aligned}
$$

Thus, (40) and (42) show that

$$
\left(e-a_{3}\right) q\left(x_{n}, x_{n+1}\right) \leq\left(a_{1}+a_{2}+a_{3}\right) q\left(x_{n-1}, x_{n}\right) .
$$

Since $r\left(a_{3}\right)<r\left(a_{3}\right)+r\left(a_{1}+a_{2}+a_{3}\right)<1$, then by Proposition 1 it follows that $e-a_{3}$ is invertible. Furthermore,

$$
\left(e-a_{3}\right)^{-1}=\sum_{i=0}^{\infty} a_{3}^{i}
$$

Let $h=\left(e-a_{3}\right)^{-1}\left(a_{1}+a_{2}+a_{3}\right)$. Since $a_{3}$ commutes with $a_{1}+a_{2}+a_{3}$, we see

$$
\begin{aligned}
& \left(e-a_{3}\right)^{-1}\left(a_{1}+a_{2}+a_{3}\right)=\left(\sum_{i=0}^{\infty} a_{3}^{i}\right)\left(a_{1}+a_{2}+a_{3}\right) \\
& =\left(a_{1}+a_{2}+a_{3}\right)\left(\sum_{i=0}^{\infty} a_{3}^{i}\right) \\
& =\left(a_{1}+a_{2}+a_{3}\right)\left(e-a_{3}\right)^{-1},
\end{aligned}
$$

that is to say $\left(e-a_{3}\right)^{-1}$ commutes with $a_{1}+a_{2}+a_{3}$. Then by Lemmas 10 and 11, we obtain

$$
\begin{aligned}
r(h) & =r\left(\left(e-a_{3}\right)^{-1}\left(a_{1}+a_{2}+a_{3}\right)\right) \\
& \leq r\left(\left(e-a_{3}\right)^{-1}\right) r\left(a_{1}+a_{2}+a_{3}\right) \\
& \leq\left(1-r\left(a_{3}\right)\right)^{-1} r\left(a_{1}+a_{2}+a_{3}\right) \\
& =\frac{1}{1-r\left(a_{3}\right)} r\left(a_{1}+a_{2}+a_{3}\right)<1,
\end{aligned}
$$

which states that $(e-h)^{-1}=\sum_{i=0}^{\infty} h^{i}$ and $\left\|h^{n}\right\| \rightarrow 0$ as $n \rightarrow \infty$.

By multiplying in both sides of (43) by $\left(e-a_{3}\right)^{-1}$, we get

$$
\begin{aligned}
q\left(x_{n}, x_{n+1}\right) & \leq\left(e-a_{3}\right)^{-1}\left(a_{1}+a_{2}+a_{3}\right) q\left(x_{n-1}, x_{n}\right) \\
& =h q\left(x_{n-1}, x_{n}\right) \preceq \cdots \preceq h^{n} q\left(x_{0}, x_{1}\right) .
\end{aligned}
$$

Let $m>n \geq 1$. It yields that

$$
\begin{aligned}
q\left(x_{n}, x_{m}\right) \leq & q\left(x_{n}, x_{n+1}\right)+q\left(x_{n+1}, x_{n+2}\right)+\cdots \\
& +q\left(x_{m-1}, x_{m}\right) \\
\leq & \left(h^{n}+h^{n+1}+\cdots+h^{m-1}\right) q\left(x_{0}, x_{1}\right) \\
= & \left(e+h+\cdots+h^{m-n-1}\right) h^{n} q\left(x_{0}, x_{1}\right) \\
& \left(\sum_{i=0}^{\infty} h^{i}\right) h^{n} q\left(x_{0}, x_{1}\right) \\
= & (e-h)^{-1} h^{n} q\left(x_{0}, x_{1}\right) .
\end{aligned}
$$

Note that $\left\|(e-h)^{-1} h^{n} q\left(x_{0}, x_{1}\right)\right\| \leq\left\|(e-h)^{-1}\right\|\left\|h^{n}\right\| \| q\left(x_{0}\right.$, $\left.x_{1}\right) \| \rightarrow 0(n \rightarrow \infty)$; then $q\left(x_{n}, x_{m}\right) \preceq(e-h)^{-1} h^{n} q\left(x_{0}, x_{1}\right) \rightarrow$ $\theta(n \rightarrow \infty)$. By Lemma 7(3), $\left\{x_{n}\right\}$ is a Cauchy sequence in $(X, d)$. 
As $X$ is complete, there exists $u \in X$ such that $x_{n} \rightarrow$ $u$ as $n \rightarrow \infty$. By Definition 4(q3), we obtain

$$
q\left(x_{n}, u\right) \preceq(e-h)^{-1} h^{n} q\left(x_{0}, x_{1}\right),
$$

which illustrates that

$$
q\left(x_{2 n+1}, u\right) \preceq(e-h)^{-1} h^{2 n+1} q\left(x_{0}, x_{1}\right) .
$$

Now, we show that $f u=g u=u$. Substituting $x=x_{2 n+1}$, $y=u$ in (37), we get

$$
\begin{aligned}
q\left(x_{2 n+1}, g u\right)= & q\left(f x_{2 n}, g u\right) \\
\preceq & a_{1} q\left(x_{2 n}, u\right)+a_{2} q\left(x_{2 n}, f x_{2 n}\right) \\
& +a_{3} q\left(x_{2 n}, g u\right) \\
\preceq & a_{1} q\left(x_{2 n}, u\right)+a_{2} q\left(x_{2 n}, x_{2 n+1}\right) \\
& +a_{3}\left[q\left(x_{2 n}, x_{2 n+1}\right)+q\left(x_{2 n+1}, g u\right)\right],
\end{aligned}
$$

which implies that $\left(e-a_{3}\right) q\left(x_{2 n+1}, g u\right) \preceq a_{1} q\left(x_{2 n}, u\right)+\left(a_{2}+\right.$ $\left.a_{3}\right) q\left(x_{2 n}, x_{2 n+1}\right)$. Since $r\left(a_{3}\right)<1, e-a_{3}$ is invertible. So, it follows immediately from (47) and (49) that

$$
\begin{aligned}
& q\left(x_{2 n+1}, g u\right) \preceq\left(e-a_{3}\right)^{-1}\left[a_{1} q\left(x_{2 n}, u\right)\right. \\
& \left.+\left(a_{2}+a_{3}\right) q\left(x_{2 n}, x_{2 n+1}\right)\right] \preceq\left(e-a_{3}\right)^{-1} \\
& \cdot\left[a_{1}(e-h)^{-1} h^{2 n} q\left(x_{0}, x_{1}\right)\right. \\
& \left.+\left(a_{2}+a_{3}\right) h^{2 n} q\left(x_{0}, x_{1}\right)\right]=\left(e-a_{3}\right)^{-1} \\
& \cdot\left[a_{1}(e-h)^{-1}+\left(a_{2}+a_{3}\right)\right] h^{2 n} q\left(x_{0}, x_{1}\right) .
\end{aligned}
$$

Set $\alpha_{n}=(e-h)^{-1} h^{2 n+1} q\left(x_{0}, x_{1}\right)$ and $\beta_{n}=\left(e-a_{3}\right)^{-1}\left[a_{1}(e-\right.$ $\left.h)^{-1}+\left(a_{2}+a_{3}\right)\right] h^{2 n} q\left(x_{0}, x_{1}\right)$. As $r(h)<1$ and $\left\|h^{n}\right\| \rightarrow 0(n \rightarrow$ $\infty)$, we know $\alpha_{n}, \beta_{n} \rightarrow \theta(n \rightarrow \infty)$. Thus, by (50), (52), and Lemma 8 , we get that $g u=u$. By repeating the similar method processes above, we suffice to prove that $f u=u$. As a result, $f u=g u=u$, that is to say $u$ is a common fixed point of $f$ and $g$.

Next, we shall show the common fixed point is unique. Firstly, we have to prove $q(u, u)=\theta$. Making full use of (37), we know

$$
\begin{aligned}
q(u, u) & =q(f u, g u) \\
& \preceq a_{1} q(u, u)+a_{2} q(u, f u)+a_{3} q(u, g u) \\
& =\left(a_{1}+a_{2}+a_{3}\right) q(u, u),
\end{aligned}
$$

which means that $\left(e-a_{1}-a_{2}-a_{3}\right) q(u, u) \preceq \theta$. Since $r\left(a_{1}+a_{2}+a_{3}\right)<1-r\left(a_{3}\right)<1$ and $a_{1}+a_{2}+a_{3}$ is invertible, then, multiplying both sides with $\left(e-a_{1}-a_{2}-a_{3}\right)^{-1}$, we have $q(u, u)=\theta$. Secondly, if there is another common fixed point $v$, then, from (37), we get

$$
\begin{aligned}
q(v, u) & =q(f v, g u) \\
& \preceq a_{1} q(v, u)+a_{2} q(v, f v)+a_{3} q(v, g u) \\
& =\left(a_{1}+a_{3}\right) q(v, u) .
\end{aligned}
$$

As $a_{1}+a_{3} \preceq a_{1}+a_{2}+a_{3}$ and $r\left(a_{1}+a_{2}+a_{3}\right)<1-r\left(a_{3}\right)<$ 1 , it follows immediately from Lemma 12 that $q(v, u)=\theta$. Actually, by (38), we also have that

$$
q(u, v) \preceq\left(a_{1}+a_{3}\right) q(u, v) .
$$

Similar to the above proof, it is not difficult to obtain that $q(u, v)=\theta$. Thus, from Lemma $9, u=v$. The conclusion is true.

Corollary 17. Let $(X, d)$ be a complete cone metric space and the underlying solid cone $P$. Let $q$ be a c-distance on $X$. Suppose the mappings $f, g: X \rightarrow X$ satisfy generalized Lipschitz conditions:

$$
\begin{aligned}
& q(f x, g y) \preceq a_{1} q(x, y)+a_{2} q(x, f x)+a_{3} q(x, g y), \\
& q(g x, f y) \preceq a_{1} q(x, y)+a_{2} q(x, g x)+a_{3} q(x, f y),
\end{aligned}
$$

for all $x, y \in X$, where $a_{i} \in \mathbb{R}^{+}(i=1,2,3)$ and satisfy $a_{1}+a_{2}+2 a_{3}<1$. Then, $f, g$ have a unique common fixed point.

Proof. By taking $r\left(a_{i}\right)=a_{i}$ for each $a_{i} \in \mathbb{R}^{+}(i=1,2,3)$ in Theorem 16, the proof is clear.

Corollary 18. Let $(X, d)$ be a complete cone metric space over Banach algebra $\mathscr{A}$ and the underlying solid cone $P$. Let $q$ be a $c$-distance on $X$. Suppose the mappings $f, g: X \rightarrow X$ satisfy generalized Lipschitz conditions:

$$
\begin{aligned}
& q(f x, g y) \preceq a_{1} q(x, y)+a_{2} q(x, f x), \\
& q(g x, f y) \preceq a_{1} q(x, y)+a_{2} q(x, g x),
\end{aligned}
$$

for all $x, y \in X$, where $a_{i} \in P(i=1,2)$ are generalized Lipschitz constants and satisfies $r\left(a_{1}+a_{2}\right)<1$. Then, $f, g$ have a unique common fixed point.

Proof. It follows by taking $a_{3}=\theta$ in Theorem 16 .

Corollary 19. Let $(X, d)$ be a complete cone metric space over Banach algebra $\mathscr{A}$ and the underlying solid cone $P$. Let $q$ be $a$ $c$-distance on $X$. Suppose the mapping $f: X \rightarrow X$ satisfies generalized Lipschitz condition:

$$
q(f x, f y) \preceq a_{1} q(x, y)+a_{2} q(x, f x),
$$

for all $x, y \in X$, where $a_{i} \in P(i=1,2)$ are generalized Lipschitz constants and satisfies $r\left(a_{1}+a_{2}\right)<1$. Then, $f$ has a unique fixed point.

Proof. It follows by taking $a_{3}=\theta$ and $f=g$ in Theorem 16 .

Corollary 20. Let $(X, d)$ be a complete cone metric space over Banach algebra $\mathscr{A}$ and the underlying solid cone $P$. Let $q$ be $a$ $c$-distance on $X$. Suppose the mapping $f: X \rightarrow X$ satisfies generalized Lipschitz condition:

$$
q(f x, f y) \preceq a_{1} q(x, y),
$$

for all $x, y \in X$, where $a_{1} \in P$ is a generalized Lipschitz constant and satisfies $r\left(a_{1}\right)<1$. Then, $f$ has a unique fixed point. 
Proof. Set $a_{2}=a_{3}=\theta$ and $f=g$ in Theorem 16, the proof is valid.

Remark 21. Taking in Corollary 20 the $c$-distance $q: X \times$ $X \rightarrow \mathscr{A}$ defined by $q(x, y)=d(x, y)$ for all $x, y \in X$. Then, Corollary 20 corresponds to Theorem 3.1 in [19]. So, Corollary 20 improves and generalizes Theorem 3.1 in [19].

Remark 22. Comparing the main results in the literature [6, 7, 9-11, 13-17], we see that many conclusions in the literature relied strongly on either the assumption of the continuity of the mappings (Theorems 3.1 and 3.3 in [6]; Theorems 3.5 and 3.9 in [9]; Theorems 3.1 and 3.3 in [10]; Theorems 2-5 in [11]; Theorem 3.3 in [14]; Theorem 2.4 and Corollaries 2.5-2.7 in [16]; Theorems 3.1, 3.2, 3.5, and 3.6 in [17]); or the normality of the cone on $c$-distance in cone metric space (Theorems 3.2 and 3.4 in [6]; Theorems 3.2 and 3.5 in [10]; Theorem 1 and Corollaries 1-3 in [13]; Theorems 3.1-3.3 and Corollaries 3.2 and 3.4 in [15]; Theorem 2.8 in [16]; Theorem 3.3, 3.4, 3.7, and 3.8 in [17]; Theorem 2.2 in [7]). They must have one of the two. In this paper, we get the fixed point theorems, which does not require the continuity of the mappings, nor does it require the normality of the cone. We delete the two conditions at the same times. Moreover, we not only obtain the existence of fixed points, but also get their uniqueness. So, the main results in this paper are indeed more different than the standard results of cone metric spaces presented in the literature. Based on these facts, our main results are very meaningful and may offer us a useful tool for the existence and uniqueness of fixed points.

Remark 23. C-distance is great generalization of cone metric ([7]). Further, due to the nonequivalence of cone metric spaces with Banach algebra and (general) metric spaces, the conclusions in this paper are focused on fixed point theorems in cone metric spaces over Banach algebras instead of theorems only in usual metric spaces or cone metric spaces (see $[1-4,6-8,10-12,14-17,21,22]$ ), which is more meaningful. If $E=\mathbb{R}$ in these theorems, we obtain the corresponding (common) fixed point theorems in metric spaces and generalize many of the conclusions in [8].

As an application, we will give an example of the situation when Theorem 13 can be applied, while the results in the literature cannot.

Example 24. Let $\mathscr{A}=C_{\mathbb{R}}^{1}[0,1] \times C_{\mathbb{R}}^{1}[0,1]$ with the norm

$$
\left\|\left(x_{1}, x_{2}\right)\right\|=\left\|x_{1}\right\|_{\infty}+\left\|x_{2}\right\|_{\infty}+\left\|x_{1}^{\prime}\right\|_{\infty}+\left\|x_{2}^{\prime}\right\|_{\infty} .
$$

Define the multiplication by

$$
x y=\left(x_{1}, x_{2}\right)\left(y_{1}, y_{2}\right)=\left(x_{1} y_{1}, x_{1} y_{2}+x_{2} y_{1}\right),
$$

where $x=\left(x_{1}, x_{2}\right), y=\left(y_{1}, y_{2}\right) \in \mathscr{A}$. Then $\mathscr{A}$ is a Banach algebra with a unit $e=(1,0)$. Let $P=\left\{\left(x_{1}(t), x_{2}(t)\right) \epsilon\right.$ $\left.\mathscr{A}: x_{1}(t) \geq 0, x_{2}(t) \geq 0, t \in[0,1]\right\}$. It is clear that $P$ is a nonnormal cone. Let $X=[0, \infty) \times[0, \infty)$ and define the cone metric $d: X \times X \rightarrow \mathscr{A}$ by $d\left(\left(x_{1}, x_{2}\right),\left(y_{1}, y_{2}\right)\right)=$ $\left(\left|x_{1}-y_{1}\right|,\left|x_{2}-y_{2}\right|\right) e^{t} \in P, \forall x=\left(x_{1}, x_{2}\right), y=\left(y_{1}, y_{2}\right) \in X$. We make a conclusion that $(X, d)$ is a complete cone metric space over Banach algebra $\mathscr{A}$. Putting a mapping $q: X \times X \rightarrow \mathscr{A}$ as $q(x, y)=y e^{t}$, it is easy to show that $q$ is a $c$-distance. Take $a_{1}=(1 / 3,1 / 6), a_{2}=(1 / 12,1 / 9), a_{3}=(1 / 6,1 / 3)$, $a_{4}=(1 / 12,1 / 6)$. We observe that

$$
r\left(a_{3}\right)+r\left(a_{1}+a_{2}+a_{3}+2 a_{4}\right)=\frac{11}{12}<1 .
$$

Furthermore, define the mapping $f: X \rightarrow X$ by

$$
f x= \begin{cases}\frac{x}{4}, & x \in \mathbb{Q} \cap X \\ \frac{x}{3}, & \text { otherwise }\end{cases}
$$

where $x \in \mathbb{Q} \cap X$ is equivalent to $x=\left(x_{1}, x_{2}\right) \in \mathbb{Q} \cap[0, \infty) \times$ $\mathbb{Q} \cap[0, \infty)$. Obviously, $f$ is not continuous. There are the following two cases.

(i) $\forall x \in X, \forall y \in \mathbb{Q} \cap X$,

$$
\begin{aligned}
q(f x, f y)= & f y \cdot e^{t}=\frac{y}{4} \cdot e^{t}=\left(\frac{y_{1}}{4}, \frac{y_{2}}{4}\right) \cdot e^{t} \\
\preceq & \left(\frac{11}{24} y_{1}, \frac{11}{24} y_{2}+\frac{5}{12} y_{1}\right) \cdot e^{t} \\
= & {\left[\left(\frac{5}{12}, \frac{1}{3}\right)\left(y_{1}, y_{2}\right)+\left(\frac{1}{6}, \frac{1}{3}\right)\left(\frac{y_{1}}{4}, \frac{y_{2}}{4}\right)\right] } \\
& \cdot e^{t}=\left[\left(a_{1}+a_{4}\right) y+a_{3} \cdot \frac{y}{4}\right] \cdot e^{t} \\
\preceq & \left(a_{1} y+a_{2} \cdot \frac{x}{4}+a_{3} \cdot \frac{y}{4}+a_{4} y\right) \cdot e^{t} \\
= & a_{1} q(x, y)+a_{2} q(x, f x)+a_{3} q(x, f y) \\
& +a_{4} q(f x, y)
\end{aligned}
$$

$\forall x \in X, \forall y \in X /(\mathbb{Q} \cap X)$,

$$
\begin{aligned}
q(f x, f y)= & f y \cdot e^{t}=\frac{y}{3} \cdot e^{t}=\left(\frac{y_{1}}{3}, \frac{y_{2}}{3}\right) \cdot e^{t} \\
\preceq & \left(\frac{17}{36} y_{1}, \frac{17}{36} y_{2}+\frac{4}{9} y_{1}\right) \cdot e^{t} \\
= & {\left[\left(\frac{5}{12}, \frac{1}{3}\right)\left(y_{1}, y_{2}\right)+\left(\frac{1}{6}, \frac{1}{3}\right)\left(\frac{y_{1}}{3}, \frac{y_{2}}{3}\right)\right] } \\
& \cdot e^{t}=\left[\left(a_{1}+a_{4}\right) y+a_{3} \cdot \frac{y}{3}\right] \cdot e^{t} \\
\preceq & \left(a_{1} y+a_{2} \cdot \frac{x}{3}+a_{3} \cdot \frac{y}{3}+a_{4} y\right) \cdot e^{t} \\
= & a_{1} q(x, y)+a_{2} q(x, f x)+a_{3} q(x, f y) \\
& +a_{4} q(f x, y) .
\end{aligned}
$$

(ii) $\forall y \in X, \forall x \in \mathbb{Q} \cap X$,

$$
\begin{aligned}
& q(f y, f x)=f x \cdot e^{t}=\frac{x}{4} \cdot e^{t}=\left(\frac{x_{1}}{4}, \frac{x_{2}}{4}\right) \cdot e^{t} \\
& \preceq\left(\frac{29}{48} x_{1}, \frac{29}{48} x_{2}+\frac{47}{72} x_{1}\right) \cdot e^{t}
\end{aligned}
$$




$$
\begin{aligned}
= & {\left[\left(\frac{7}{12}, \frac{11}{18}\right)\left(x_{1}, x_{2}\right)+\left(\frac{1}{12}, \frac{1}{6}\right)\left(\frac{x_{1}}{4}, \frac{x_{2}}{4}\right)\right] \cdot e^{t} } \\
= & {\left[\left(a_{1}+a_{2}+a_{3}\right) x+a_{4} \cdot \frac{x}{4}\right] \cdot e^{t} } \\
= & \left(a_{1} x+a_{2} x+a_{3} x+a_{4} \cdot \frac{x}{4}\right) \cdot e^{t} \\
= & a_{1} q(y, x)+a_{2} q(f x, x)+a_{3} q(f y, x) \\
& +a_{4} q(y, f x) ;
\end{aligned}
$$

$\forall y \in X, \forall x \in X /(\mathbb{Q} \cap X)$,

$$
\begin{aligned}
q( & f y, f x)=f x \cdot e^{t}=\frac{x}{3} \cdot e^{t}=\left(\frac{x_{1}}{3}, \frac{x_{2}}{3}\right) \cdot e^{t} \\
\leq & \left(\frac{11}{18} x_{1}, \frac{11}{18} x_{2}+\frac{2}{3} x_{1}\right) \cdot e^{t} \\
= & {\left[\left(\frac{7}{12}, \frac{11}{18}\right)\left(x_{1}, x_{2}\right)+\left(\frac{1}{12}, \frac{1}{6}\right)\left(\frac{x_{1}}{3}, \frac{x_{2}}{3}\right)\right] \cdot e^{t} } \\
= & {\left[\left(a_{1}+a_{2}+a_{3}\right) x+a_{4} \cdot \frac{x}{3}\right] \cdot e^{t} } \\
= & \left(a_{1} x+a_{2} x+a_{3} x+a_{4} \cdot \frac{x}{3}\right) \cdot e^{t} \\
= & a_{1} q(y, x)+a_{2} q(f x, x)+a_{3} q(f y, x) \\
& +a_{4} q(y, f x) .
\end{aligned}
$$

Therefore, we can apply Theorem 13 and conclude that $f$ has a (unique) fixed point 0 in $X$. Since $f$ is not continuous in $X$ and the cone is not normal, we can conclude that these known results in the literature cannot cope with Example 24, which shows that the main results in our paper are actual generalization of the known results.

\section{Data Availability}

No data were used to support this study.

\section{Conflicts of Interest}

The authors declare that there are no conflicts of interest regarding the publication of this paper.

\section{Acknowledgments}

The research was partially supported by Yunnan Applied Basic Research Projects, Yunnan Province, China (no. 2016FD082).

\section{References}

[1] L. Huang and X. Zhang, "Cone metric spaces and fixed point theorems of contractive mappings," Journal of Mathematical Analysis and Applications, vol. 332, no. 2, pp. 1468-1476, 2007.
[2] S. Rezapour and R. Hamlbarani, "Some notes on the paper "Cone metric spaces and fixed point theorems of contractive mappings"," Journal of Mathematical Analysis and Applications, vol. 345, no. 2, pp. 719-724, 2008.

[3] M. Abbas and B. Rhoades, "Fixed and periodic point results in cone metric spaces," Applied Mathematics Letters, vol. 22, no. 4, pp. 511-515, 2009.

[4] H. K. Nashie, Y. Rohen, and C. Thokchom, "Common coupled fixed point theorems of two mappings satisfying generalized contractive condition in cone metric space," International Journal of Pure and Applied Mathematics, vol. 106, no. 3, pp. 791-799, 2016.

[5] H. Liu and S. Xu, "Cone metric spaces with Banach algebras and fixed point theorems of generalized Lipschitz mappings," Fixed Point Theory and Applications, vol. 2013, article no. 320, 2013.

[6] Y. J. Cho, R. Saadati, and S. H. Wang, "Common fixed point theorems on generalized distance in ordered cone metric spaces," Computers \& Mathematics with Applications, vol. 61, no. 4, pp. 1254-1260, 2011.

[7] S. Wang and B. Guo, "Distance in cone metric spaces and common fixed point theorems," Applied Mathematics Letters, vol. 24, no. 10, pp. 1735-1739, 2011.

[8] Q. Kada, S. Suzuki, and W. Takahashi, "Nonconvex minimization theorems and fixed point theorems in complete metric spaces," Mathematica Japonica, vol. 44, pp. 381-391, 1996.

[9] Q. Yan, J. Yin, and T. Wang, "Fixed point and common fixed point theorems on ordered cone metric spaces over Banach algebras," Journal of Nonlinear Science and Applications, vol. 9, no. 4, pp. 1581-1589, 2016.

[10] W. Sintunavarat, Y. J. Cho, and P. Kumam, "Common fixed point theorems for c-distance in ordered cone metric spaces," Computers \& Mathematics with Applications, vol. 62, no. 4, pp. 1969-1978, 2011.

[11] M. Đorđević, D. Đorić, Z. Kadelburg, S. Radenović, and D. Spasić, "Fixed point results under c-distance in tvs-cone metric spaces," Fixed Point Theory and Applications, vol. 2011, article 29, 2011.

[12] H. Aydi, E. Karapinar, and S. Moradi, "Coincidence points for expansive mappings under c-distance in cone metric spaces," International Journal of Mathematics and Mathematical Sciences, vol. 2012, Article ID 308921, 2012.

[13] H. Huang, S. Radenović, and T. Došenović, "Some common fixed point theorems on c-distance in cone metric spaces over banach algebras," Applied and Computational Mathematics, vol. 14, no. 2, pp. 180-193, 2015.

[14] Z. M. Fadail and A. G. Bin Ahmad, "Common coupled fixed point theorems of single-valued mapping for c-distance in cone metric spaces," Abstract and Applied Analysis, vol. 2012, Article ID 901792, 2012.

[15] H. Rahimi, G. Soleimani Rad, and P. Kumam, "Generalized distance and new fixed point results," Asian-European Journal of Mathematics, vol. 9, no. 2, Article ID 1650044, 2016.

[16] Y.-O. Yang and H. J. Choi, "Fixed point theorems in ordered cone metric spaces," Journal of Nonlinear Science and Applications, vol. 9, no. 6, pp. 4571-4579, 2016.

[17] H. Rahimi and G. S. Rad, "Common fixed-point theorems and c-distance in ordered cone metric spaces," Ukrainian Mathematical Journal, vol. 65, no. 12, pp. 1845-1861, 2014.

[18] W. Rudin, Functional Analysis, McGraw-Hill, New York, NY, USA, 2nd edition, 1991. 
[19] S. Xu and S. Radenović, "Fixed point theorems of generalized Lipschitz mappings on cone metric spaces over Banach algebras without assumption of normality," Fixed Point Theory and Applications, vol. 2014, no. 1, p. 102, 2014.

[20] H. Huang, S. Hu, B. Z. Popović, and S. Radenović, "Common fixed point theorems for four mappings on cone b-metric spaces over Banach algebras," Journal of Nonlinear Science and Applications, vol. 9, no. 6, pp. 3655-3671, 2016.

[21] Z. Kadelburg, M. Pavlović, and S. Radenović, "Common fixed point theorems for ordered contractions and quasicontractions in ordered cone metric spaces," Computers \& Mathematics with Applications, vol. 59, no. 9, pp. 3148-3159, 2010.

[22] I. Altun and G. Durmaz, "Some fixed point theorems on ordered cone metric spaces," Rendiconti del Circolo Matematico di Palermo , vol. 58, no. 2, pp. 319-325, 2009. 


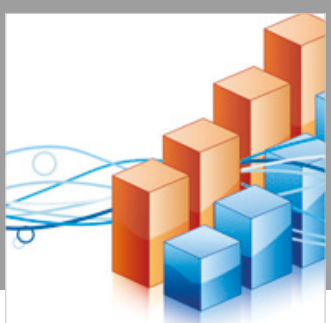

Advances in

Operations Research

\section{-n-m}
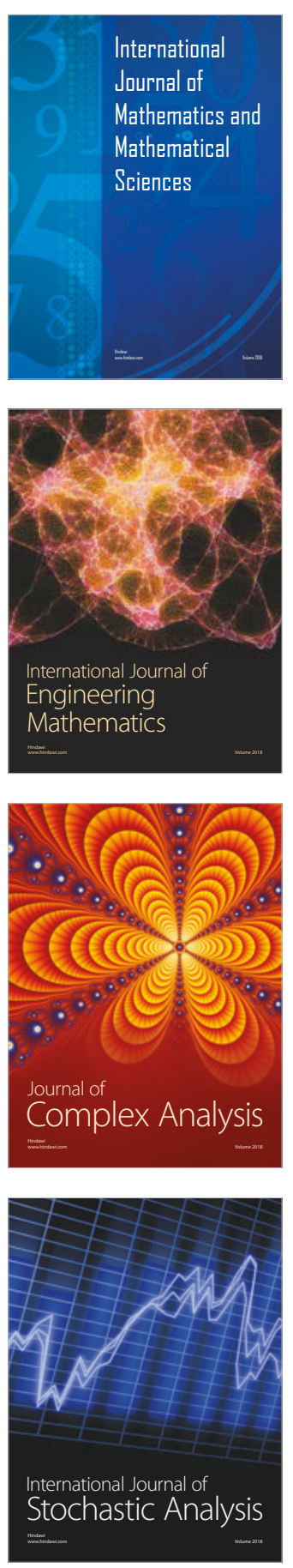
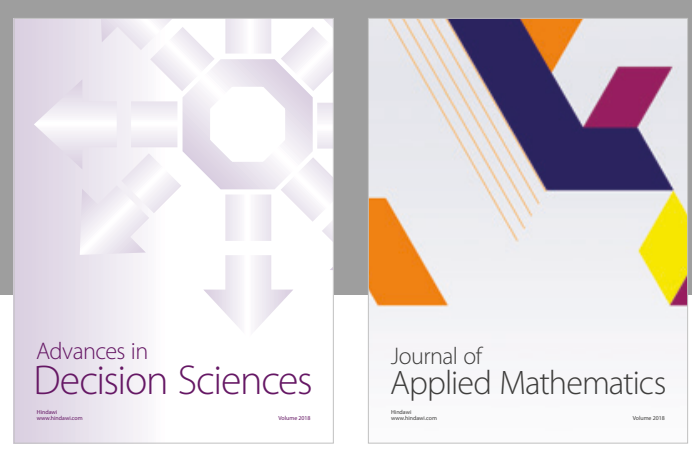

Journal of

Applied Mathematics
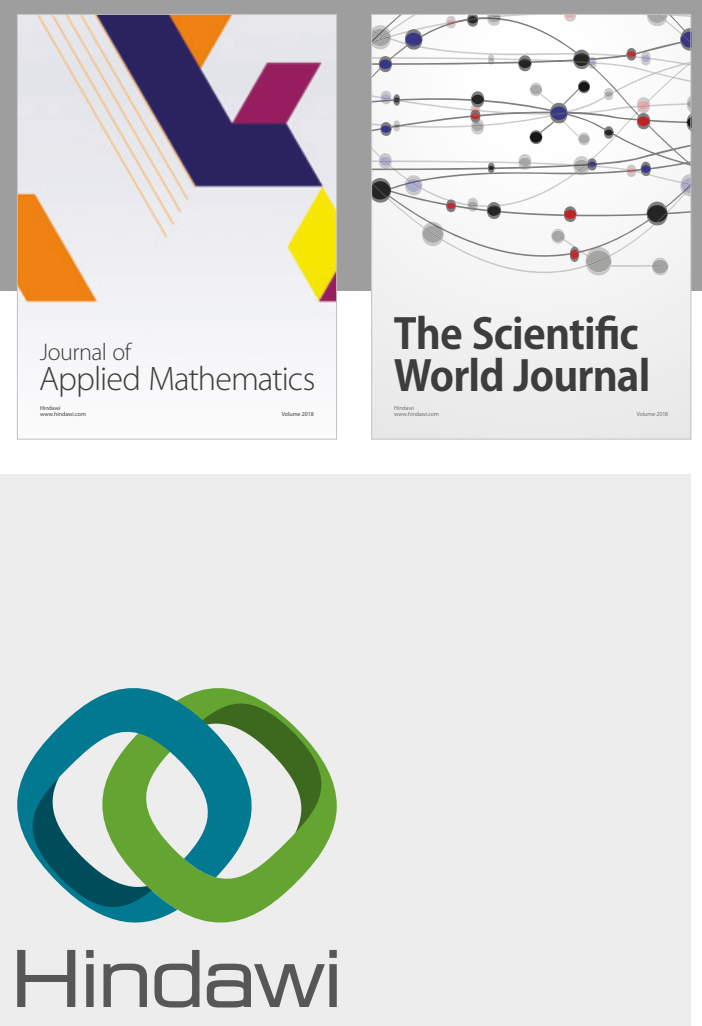

Submit your manuscripts at

www.hindawi.com

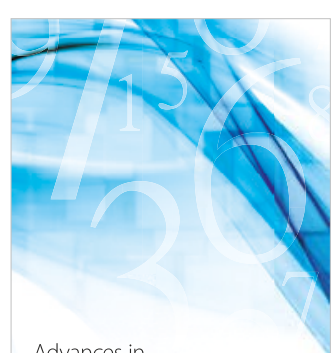

Advances in
Numerical Analysis
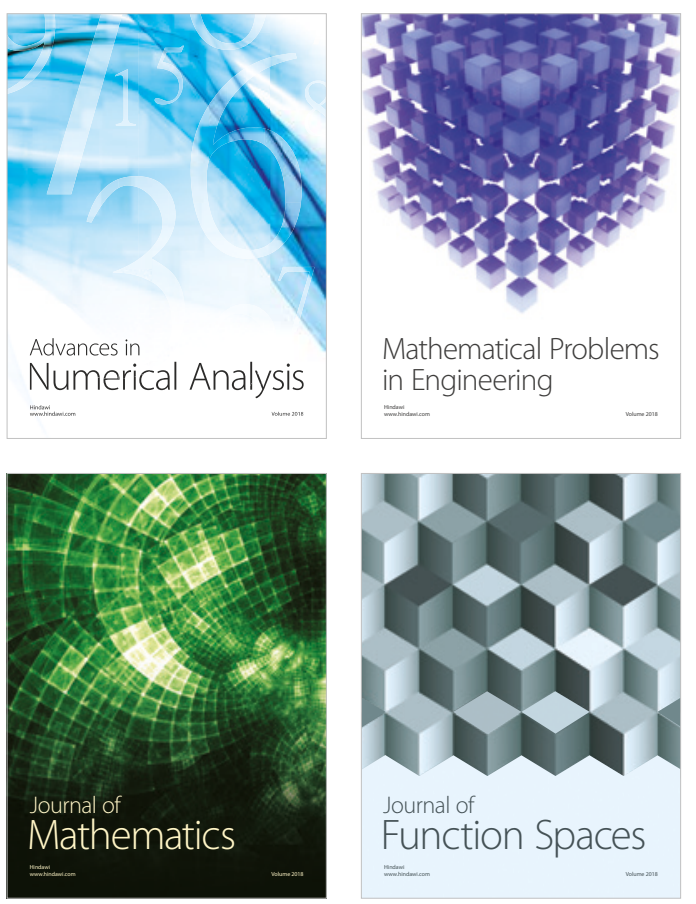

Mathematical Problems in Engineering

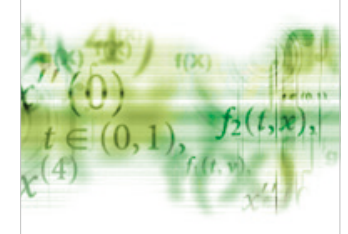

International Journal of

Differential Equations

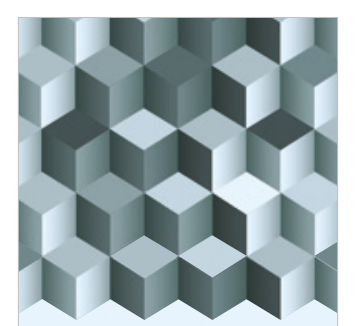

Journal of

Function Spaces

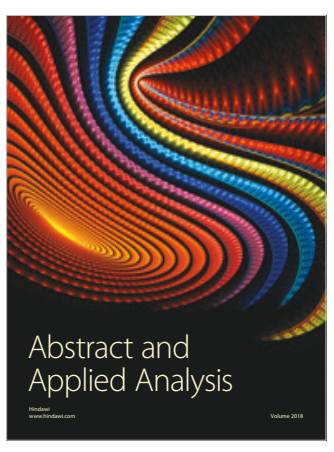

The Scientific

World Journal

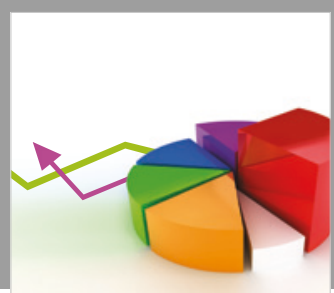

Journal of

Probability and Statistics
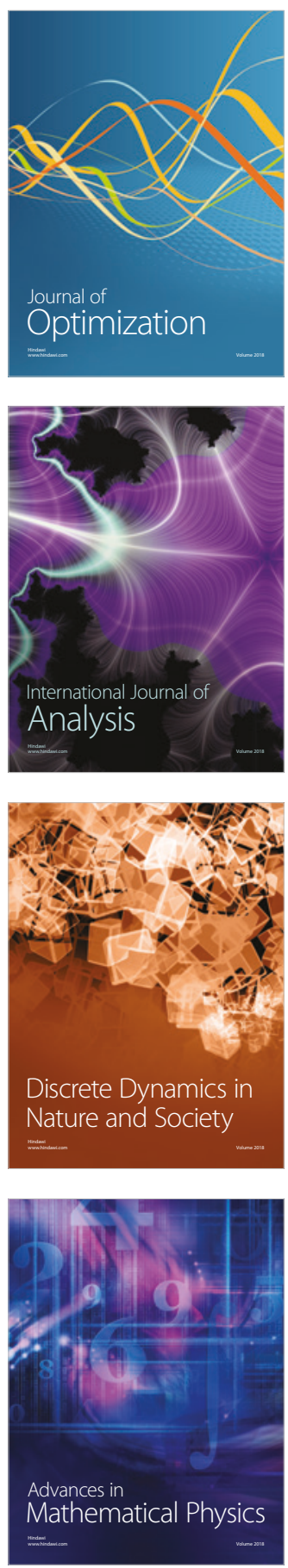